\title{
Stellar Activity with BRITE: the "Aurigae" field
}

\author{
K. G. Strassmeier \\ Astrophysical Institute Potsdam (AIP) \\ An der Sternwarte 16, D-14482 Potsdam, Germany
}

\section{Abstract}

Photometric time series of active stars can pin down some of the ingredients that govern the stellar magnetic field, itself being the driver of all non-thermal stellar emissions. Among the most important - and least understood - astrophysical ingredients is stellar rotation and its subtle latitudinal dependence called differential rotation. Rotation switches on and maintains the internal dynamo, itself a phenomenon from the interaction of turbulent plasma motions and large-scale shearing forces in the deep stellar interior. I propose to observe the active binary Capella, made up of two giants in exposed locations in the HR-diagram. Along with Capella ( $\alpha$ Aur), another eight stars brighter than 4th magnitude would be in the field-of-view of BRITE, among them such benchmark variables as $\zeta$ Aur or $\theta$ Aur.

\section{Motivation}

The interaction of a stellar magnetic field with its surrounding plasma appears to be the source of a large variety of atmospheric phenomena that can be used to better understand a star and its evolution itself as well as study its environment. Such magnetic-field related phenomena were collectively coined "stellar activity". Our magnetic Sun and the many non-thermal energetic phenomena from its interaction with the outer heliosphere, also known as "space weather", play even a central role for our own planet. Therefore, stellar-activity studies continuously moved central stage after the discovery of extra-solar planets back in the mid nineties. The reason is the many connections between the parent star's output and the impact on its planetary system, from the time of its formation until its habitability.

Magnetic fields brake the rotation of the collapsing pre-stellar cloud enabling star formation in the first place and, later, couple the rotation of the star with its pre-planetary disk. This is a violent time for the star. But even much later, at the age of the Sun, its magnetic field still emits energetic radiation 
conducive to the destruction of complex molecules in the upper Earth atmosphere. It also governs the habitability of the biosphere through this energetic particle bombardment. The Earths magnetic field again protects us from it. Understanding solar and stellar magnetic activity likely holds the key to glimpse into the galaxy's biological evolution, if there is one. The search for Earth-like planets is part of this enterprize. Therefore, we believe that studying the rotation of (low-mass) stars that harbor significant magnetic fields, i.e. active stars, provides the physics for such illuminative questions as whether life could exist elsewhere in the universe.

\section{Stellar activity and photometry}

A magnetically-active star will show variations of its brightness because dark spots or bright plages will move in an out of view as the star rotates. It enables the observation of very precise stellar rotational periods and, coupled with knowledge of its radius, the determination of the star's specific angular momentum; for low-mass stars at certain stages in their evolution this is a rather important physical quantity. Monitoring the brightness variations over many consecutive stellar rotations can be used to infer the surface spot and plage evolution, itself being just localized magnetic flux. Modern mathematical inversion methods allow the recovery of the two-dimensional surface structure from individual light curves (e.g. Savanov \& Strassmeier 2007, among others), given that they can be obtained nearly uninterrupted.

Furthermore, one can isolate the rotational period for each latitudinal strip on the surface at which a spot or a plage appears, thereby detecting and measuring differential surface rotation, one of the two main ingredients for a stellar dynamo (the other is helicity).

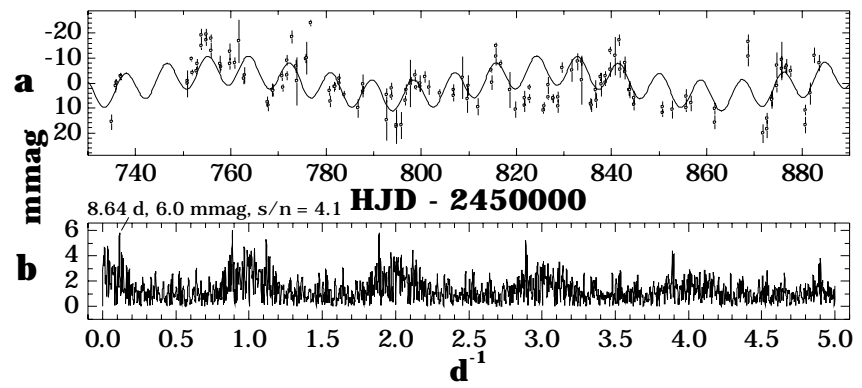

Figure 1: a The $\mathrm{H} \alpha$-light curve of Capella during the observing season 1997/98 (dots with error bars) and the fit from a two-frequency solution with a 106-day and a 8.6-day period. $\mathbf{b}$ The amplitude spectrum for the above data. 


\section{Is BRITE relevant for active-star photometry?}

Yes, even given the brightness limit of 4th magnitude for 1-mmag precision, but being a bit generous and expanding this to 5th magnitude, we find a total of 17 active stars in the sky. All of them are evolved stars. The brightest five (all brighter than $4.2 \mathrm{mag}$ ) are luminosity-class III giants, again all of them binaries and one even in an eclipsing system. Only two are single Hertzsprung-gap ${ }^{1}$ giants. In this respect, BRITE's golden bullet would be to monitor an active giant's rotationally modulated light curve for a long duration in time.

\section{What you usually can't do from the ground}

Observing bright stars with small amplitudes from the ground is a painstaking challenge, if possible at all. Fig. 1 shows our photometry of the brightest active star in the sky, $V=0.1$-mag Capella ( $\alpha$ Aurigae). This data was taken with one of the two $0.75 \mathrm{~m}$ Vienna APTs equipped with a 5-mag neutral density filter and a narrow-band $\mathrm{H} \alpha$ filter. We believe we have detected the rotational periods of both stellar components of Capella (Strassmeier et al. 2001). The brighter $\mathrm{K}$ giant appears to rotate with 106 days and the fainter but more active early-G giant with 8.6 days. However, the quality of the data is such that these periods have large error bars or can even be questioned but, what is more important, the Capella system appears to be an important laboratory for testing tidal friction theories - synchronization, circularization and coplanarity - in combination with stellar evolution theory.

The Capella system: what's so interesting?

Astrophysical parameters of giant stars are uncertain because there are just a few such stars that can be studied in spectroscopic binary systems, and even less that are also eclipsing binaries. Roche lobe overflow and mass exchange set a limit to the stellar radius in a close binary. Non-interacting binaries with giant components have therefore mostly very long orbital periods and are difficult to observe within an astronomer's lifetime. There are, however, a few systems that have sufficiently short orbital periods (see, e.g., Andersen 1991). Capella is such a system and consists of an active G1III and a G8-KOIII component (Strassmeier \& Fekel 1990) in a 104-day orbit.

The present position of the G1III component in the Hertzsprung gap, where it is approaching the base of the giant branch, indicates violent changes are taking place in its internal structure; the mass of the convection zone increases rapidly as does also the total stellar moment of inertia. We may expect that

\footnotetext{
${ }^{1}$ When crossing the Hertzsprung gap the star quickly redistributes internal angular momentum and causes increased surface rotation for a brief period in time.
} 
this has a profound impact on the visible surface rotation. Precise rotation periods of stars in this evolutionary stage may thus help to further understand the angular-momentum loss in late-type stars.

Shcherbakov et al. (1990) discovered a modulation of the He I $1.08 \mu$ m equivalent width with the 104-day orbital period. This variation, and its periodicity, was later confirmed by Katsova \& Shcherbakov (1998). The latter authors also concluded that the main He I-absorption line must originate in the chromosphere of the quiescent cool component, while the residual helium emission and the highly ionized iron lines are thought to be connected with a magnetized wind that originates from plages on the active hot component and then forms a shock wave in the corona of the cool component. Therefore, chromospheric emission is expected from both components.

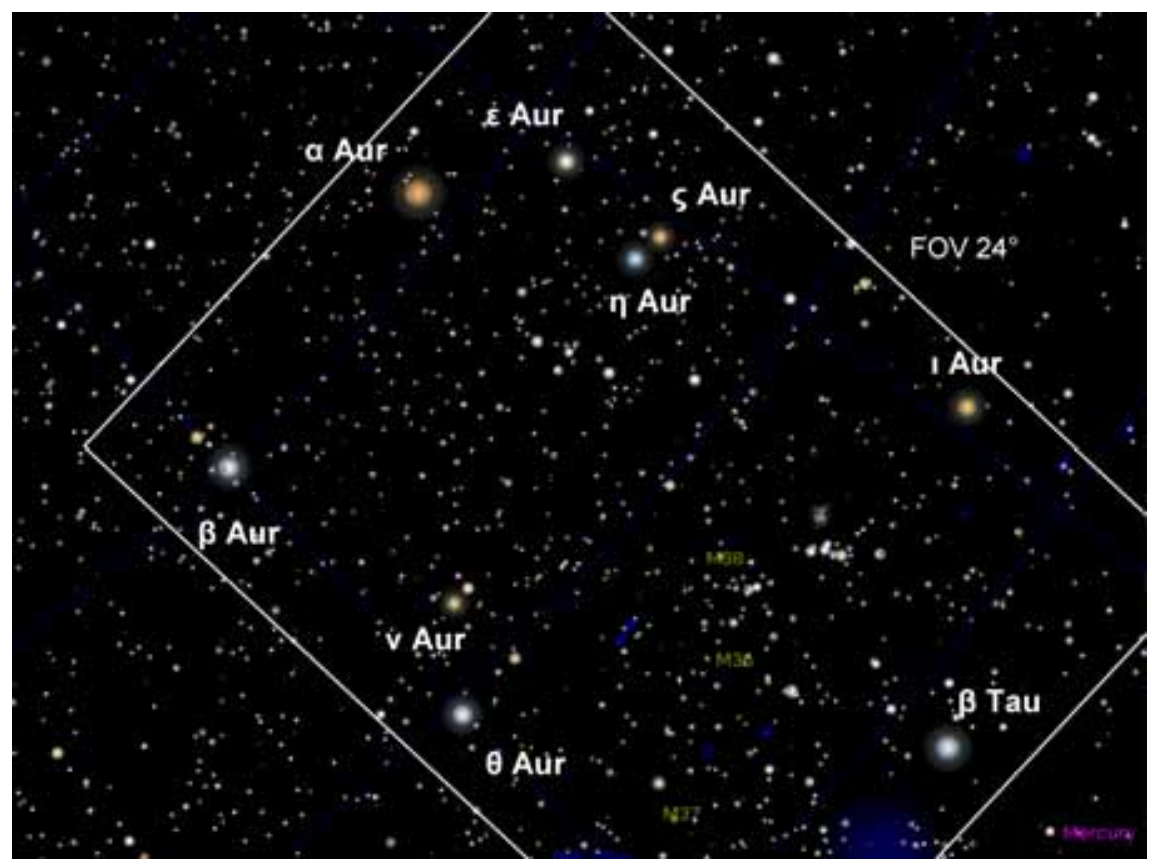

Figure 2: The proposed "Aurigae" field. It contains nine variable stars brighter than 4th magnitude. The brightest being the active binary Capella but each one represents a distinctive class of its own with a large variety of astrophysical challenges. 


\section{Conclusion: observe a 24-degree field in Aurigae}

My proposal is to dedicate BRITE time to Capella. Sampling requirement would be one measurement per satellite orbit, but at least one every 1-2 hours, for a total duration of $>104$ days (the orbital period). Comparably short exposures and on-board coadding of many CCD frames would be required. Simultaneous Doppler imaging of the G-giant during the two quadrature phases with our ground-based facility STELLA/SES could be provided and would make up a unique data set. Additionally, the Capella position in the sky is very favorable for catching other, bright stars at the same time, some of them famous variable stars with lots of unknown astrophysics. Table 1 is a summary of these stars.

$\beta$ Aur is a detached eclipsing binary that was used recently to determine non-linear limb-darkening coefficients for both of its components based on highprecision photometry from the WIRE satellite (Southworth et al. 2007).

$\epsilon$ Aur is a binary star system that eclipses once every 27.1 years. It is the star with the longest period known to date. The next eclipse is predicted to begin in 2009. The eclipse is flat-bottomed and lasts nearly two years. A mideclipse brightening was seen by several observers, including observations from space, and many models appeared for its explanation. Among them a neutron star with a warped semi-opaque disk. Photometry of the primary F star appears to show pulsational variability (Strassmeier et al. 2001).

$\zeta$ Aur is a long-period eclipsing binary with a $\mathrm{K}$ supergiant and a mid-B main sequence star. A recent orbit was determined by Griffin (2005) with a period of 972 days. The importance are the eclipses of the B star when it traverses the chromosphere of the $\mathrm{K}$ star, which lasts for some weeks either side of the eclipse. It has been used to probe the K supergiant's atmosphere as a function of height above the limb.

$\eta$ Aur is a chemically peculiar B3 main-sequence star. It appears to be single. Crawford et al. (1971) lists it as one of their photometric standard stars while Simbad quotes it as a Variable.

$\theta$ Aur is a well-known chemically peculiar Ap star whose surface elemental distribution had been spatially resolved by means of Doppler imaging. Theta Aur $(\mathrm{P}=3.6188 \mathrm{~d})$ exhibits large amplitude variations with two components contributing to the light minima (e.g. Adelman 1997).

$\nu$ Aur. Numerous photometric observations of this star exist but where mostly taken in the reference of standard-star all-sky photometry (e.g. Oja 1985, Eggen 1963). It is not conclusively known whether it is a micro variable or not. Simbad lists it as part of a wide binary.

$\iota$ Aur. Although a suspected variable, its light variability has not been proven so far. The star is a single K3 bright giant. Recently, Lèbre et al. (2006) determined its lithium abundance and gave a summary of its rotational 
Table 1: Stars brighter than 4th magnitude in the "Aurigae" field.

\begin{tabular}{|c|c|c|c|c|}
\hline Star & $\begin{array}{l}V \\
\text { (mag) }\end{array}$ & $\begin{array}{l}\text { Sp. } \\
\text { type }\end{array}$ & $\begin{array}{l}\text { Type of } \\
\text { variability }\end{array}$ & Notes \\
\hline$\alpha$ Aur & 0.1 & $\begin{array}{l}\text { G1III } \\
+ \text { KollI }\end{array}$ & Rotation & Binary with two giant stars \\
\hline$\beta$ Aur & 1.9 & $\mathrm{~A} 2 \mathrm{IV}+$ & Eclipsing & Detached eclipsing system \\
\hline$\epsilon$ Aur & 3.0 & Fola & $\begin{array}{l}\text { Eclipsing } \\
\text { Pulsating }\end{array}$ & $\begin{array}{l}\text { Binary with longest known } \\
\text { orbital period }\end{array}$ \\
\hline$\zeta$ Aur & 3.8 & $\begin{array}{l}\text { K4lb } \\
+\mathrm{B} 6 \mathrm{~V}\end{array}$ & Eclipsing & Atmospheric eclipses \\
\hline$\eta$ Aur & 3.1 & B3V & Rotation & Chemically peculiar B star \\
\hline$\theta$ Aur & 2.6 & AOp & Rotation & Magnetic chemically pecul. star \\
\hline$\nu$ Aur & 4.0 & KOIII & unknown & Binary \\
\hline$\iota$ Aur & 2.7 & K3II & unknown & Suspected variable \\
\hline$\beta$ Tau & 1.7 & B7III & unknown & Chemically peculiar HgMn star \\
\hline
\end{tabular}

properties.

$\beta$ Tau. Also known as $\gamma$ Aur. Again, no continuous photometry seems to exist of this very bright binary. It has received substantial attention after it was listed among the few mercury-manganese stars (see Adelman et al. 2006).

Acknowledgments. I thank Werner Weiss and Michel Breger for inviting me to this workshop. Substantial use was made of the SIMBAD data base run by CDS Strasbourg.

\section{References}

Adelman, S.J. 1997, PASP 109, 9

Adelman, S.J., Caliskan, H., Gulliver, A.F., et al. 2006, A\&A 447, 685

Andersen, J. 1991, A\&AR 3, 91

Crawford, D.L., Barnes, J.V., Golson, J.C. 1971, AJ 76, 1058

Eggen, O.J. 1963, AJ 68, 483

Griffin, R.F. 2005, The Obs. 125, 1

Katsova, M.M., Shcherbakov, A.G. 1998, A\&A 329, 1080

Lèbre, A., de Laverny, P., Do Nascimento, J.D., et al., 2006, A\&A 450, 1173

Oja, T. 1985, A\&AS 59, 461

Savanov, I., Strassmeier, K.G. 2007, A\&A, submitted

Shcherbakov, A.G., Tuominen, I., Jetsu, L., et al. 1990, A\&A 235, 205 
Southworth, J., Bruntt, H., Buzasi, D.L. 2007, A\&A 467, 1215

Strassmeier, K.G., Fekel, F.C. 1990, A\&A 230, 389

Strassmeier, K.G., Reegen, P., Granzer, T. 2001, AN 321, 115

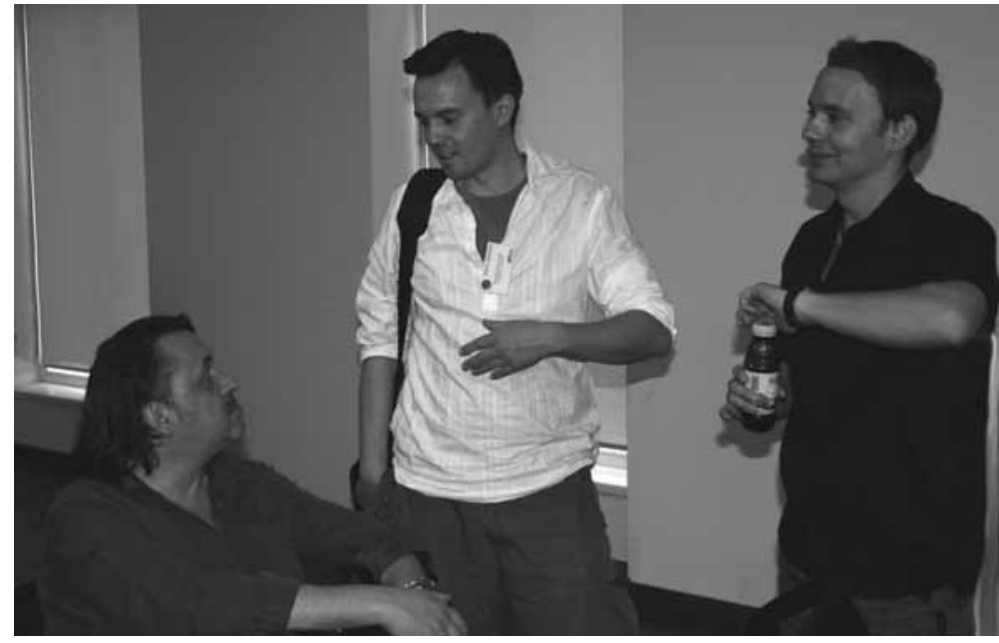

R. Kuschnig, T. Kallinger and A. Kaiser discussing the workshop. 